\title{
Some Results on Lorentzian Para-Sasakian Manifolds
}

\author{
Venkatesha, C. S. Bagewadi, and K. T. Pradeep Kumar \\ Department of Mathematics, Kuvempu University, Shankaraghatta, Karnataka, Shimoga 577 451, India
}

Correspondence should be addressed to Venkatesha, vensprem@gmail.com

Received 3 June 2011; Accepted 3 July 2011

Academic Editor: M. Dunajski

Copyright (C) 2011 Venkatesha et al. This is an open access article distributed under the Creative Commons Attribution License, which permits unrestricted use, distribution, and reproduction in any medium, provided the original work is properly cited.

The object of the present paper is to study Lorentzian para-Sasakian (briefly LP-Sasakian) manifolds satisfying certain conditions on the $W_{2}$-curvature tensor.

\section{Introduction}

In 1989, Matsumoto [1] introduced the notion of Lorentzian para-Sasakian manifold. Then, Mihai and Roşca [2] introduced the same notion independently and they obtained several results on this manifold. LP-Sasakian manifolds have also been studied by Matsumoto and Mihai [3], Mihai et al. [4], and Venkatesha and Bagewadi [5].

On the other hand, Pokhariyal and Mishra [6] have introduced new curvature tensor called $W_{2}$-curvature tensor in a Riemannian manifold and studied their properties. Further, Pokhariyal [7] has studied some properties of this curvature tensor in a Sasakian manifold. Matsumoto et al. [8], and Yìldìz and De [9] have studied $W_{2}$-curvature tensor in P-Sasakian and Kenmotsu manifolds, respectively.

In the present paper, we study some curvature conditions on LP-Sasakian manifolds. Firstly, we study LP-Sasakian manifolds satisfying $W_{2}=0$ and $W_{2}$-semisymmetric LPSasakian manifolds. Further, we study LP-Sasakian manifolds which satisfy $P \cdot W_{2}=0$, $\widetilde{M} \cdot W_{2}=0$ and $C \cdot W_{2}=0$, where $P$ is the projective curvature tensor, $\widetilde{M}$ is the $M$-projective curvature tensor, and $C$ is the conformal curvature tensor.

\section{Preliminaries}

An $n$-dimensional differentiable manifold $M$ is called an LP-Sasakian manifold $[1,2]$ if it admits a $(1,1)$ tensor field $\phi$, a contravariant vector field $\xi$, a 1-form $\eta$, and a Lorentzian 
metric $g$ which satisfy

$$
\begin{gathered}
\eta(\xi)=-1, \\
\phi^{2} X=X+\eta(X) \xi \\
g(\phi X, \phi Y)=g(X, Y)+\eta(X) \eta(Y), \\
g(X, \xi)=\eta(X), \\
\nabla_{X} \xi=\phi X, \\
\left(\nabla_{X} \phi\right) Y=g(X, Y) \xi+\eta(Y) X+2 \eta(X) \eta(Y) \xi,
\end{gathered}
$$

where $\nabla$ denotes the operator of covariant differentiation with respect to the Lorentzian metric $g$.

It can be easily seen that, in an LP-Sasakian manifold, the following relations hold:

$$
\phi \xi=0, \quad \eta(\phi X)=0, \quad \operatorname{rank} \phi=n-1 .
$$

If we put

$$
\Phi(X, Y)=g(X, \phi Y)
$$

for any vector fields $X$ and $Y$, then the tensor field $\Phi(X, Y)$ is a symmetric $(0,2)$ tensor field [1]. Also, since the 1-form $\eta$ is closed in an LP-Sasakian manifold, we have $[1,4]$

$$
\left(\nabla_{X} \eta\right)(Y)=\Phi(X, Y), \quad \Phi(X, \xi)=0,
$$

for any vector fields $X$ and $Y$.

Also in an LP-Sasakian manifold, the following relations hold [3, 4]:

$$
\begin{gathered}
g(R(X, Y) Z, \xi)=\eta(R(X, Y) Z)=g(Y, Z) \eta(X)-g(X, Z) \eta(Y) \\
R(\xi, X) Y=g(X, Y) \xi-\eta(Y) X \\
R(X, Y) \xi=\eta(Y) X-\eta(X) Y \\
S(X, \xi)=(n-1) \eta(X) \\
S(\phi X, \phi Y)=S(X, Y)+(n-1) \eta(X) \eta(Y)
\end{gathered}
$$


for any vector fields $X, Y$, and $Z$, where $R$ is the Riemannian curvature tensor and $S$ is the Ricci tensor of $M$.

An LP-Sasakian manifold $M$ is said to be Einstein if its Ricci tensor $S$ is of the form

$$
S(X, Y)=\operatorname{ag}(X, Y)
$$

for any vector fields $X$ and $Y$, where $a$ is a function on $M$.

In [6], Pokhariyal and Mishra have defined the curvature tensor $W_{2}$, given by

$$
W_{2}(X, Y, U, V)=R(X, Y, U, V)+\frac{1}{n-1}[g(X, U) S(Y, V)-g(Y, U) S(X, V)]
$$

where $S$ is a Ricci tensor of type $(0,2)$.

Now, consider an LP-Sasakian manifold satisfying $W_{2}=0$; then, (2.11) becomes

$$
R(X, Y, U, V)=\frac{1}{n-1}[g(Y, U) S(X, V)-g(X, U) S(Y, V)]
$$

Taking $X=U=\xi$ in (2.12) and using (2.7) and (2.8), we have

$$
S(Y, V)=(n-1) g(Y, V)
$$

Therefore, $M$ is an Einstein manifold.

Again using (2.13) in (2.12), we get

$$
R(X, Y, U, V)=[g(Y, U) g(X, V)-g(X, U) g(Y, V)]
$$

Corollary 2.1. An LP-Sasakian manifold satisfying $W_{2}=0$ is a space of constant curvature -1 , that is, it is locally isometric to the hyperbolic space.

Definition 2.2. An LP-Sasakian manifold is called $W_{2}$-semisymmetric if it satisfies

$$
R(X, Y) \cdot W_{2}=0
$$

where $R(X, Y)$ is to be considered as a derivation of the tensor algebra at each point of the manifold for tangent vectors $X$ and $Y$.

It can be easily shown that in an LP-Sasakian manifold the $W_{2}$-curvature tensor satisfies the condition

$$
\eta\left(W_{2}(X, Y) Z\right)=0
$$


Theorem 2.3. $A W_{2}$-semisymmetric LP-Sasakian manifold $M$ is an Einstein manifold.

Proof. Since $R(X, Y) \cdot W_{2}=0$, we have

$$
\begin{aligned}
& R(X, Y) W_{2}(U, V) Z-W_{2}(R(X, Y) U, V) Z \\
& \quad-W_{2}(U, R(X, Y) V) Z-W_{2}(U, V) R(X, Y) Z=0 .
\end{aligned}
$$

Putting $X=\xi$ in (2.17) and then taking the inner product with $\xi$, we obtain

$$
\begin{aligned}
& g\left(R(\xi, Y) W_{2}(U, V) Z, \xi\right)-g\left(W_{2}(R(\xi, Y) U, V) Z, \xi\right) \\
& \quad-g\left(W_{2}(U, R(\xi, Y) V) Z, \xi\right)-g\left(W_{2}(U, V) R(\xi, Y) Z, \xi\right)=0 .
\end{aligned}
$$

Using (2.6) in (2.18), we obtain

$$
\begin{aligned}
- & g\left(Y, W_{2}(U, V) Z\right)-\eta\left(W_{2}(U, V) Z\right) \eta(Y)-g(Y, U) \eta\left(W_{2}(\xi, V) Z\right) \\
& -g(Y, V) \eta\left(W_{2}(U, \xi) Z\right)-g(Y, Z) \eta\left(W_{2}(U, V) \xi\right)+\eta(U) \eta\left(W_{2}(Y, V) Z\right) \\
& +\eta(V) \eta\left(W_{2}(U, Y) Z\right)+\eta(Z) \eta\left(W_{2}(U, V) Y\right)=0 .
\end{aligned}
$$

By using (2.16) in (2.19), we get

$$
W_{2}(U, V, Z, Y)=0
$$

In view of (2.11) and (2.20), it follows that

$$
R(U, V, Z, Y)=\frac{1}{n-1}[g(V, Z) S(U, Y)-g(U, Z) S(V, Y)]
$$

Contracting (2.21), we have

$$
S(V, Z)=(n-1) g(V, Z)
$$

Therefore, $M$ is an Einstein manifold.

Again using (2.22) in (2.12), we get

$$
R(U, V, Z, Y)=[g(V, Z) g(U, Y)-g(U, Z) g(V, Y)]
$$

Corollary 2.4. A $W_{2}$-semisymmetric LP-Sasakian manifold is a space of constant curvature -1, that is, it is locally isometric to the hyperbolic space. 
3. LP-Sasakian Manifolds Satisfying $P(X, Y) \cdot W_{2}=0$

The projective curvature tensor $P$ is defined as [10]

$$
P(X, Y) Z=R(X, Y) Z-\frac{1}{n-1}[S(Y, Z) X-S(X, Z) Y]
$$

Using (2.6) and (2.8), (3.1) reduces to

$$
P(\xi, Y) Z=g(Y, Z) \xi-\frac{1}{n-1} S(Y, Z) \xi
$$

Let us suppose that in an LP-Sasakian manifold

$$
P(X, Y) \cdot W_{2}=0
$$

This condition implies that

$$
\begin{aligned}
& P(X, Y) W_{2}(U, V) Z-W_{2}(P(X, Y) U, V) Z \\
& \quad-W_{2}(U, P(X, Y) V) Z-W_{2}(U, V) P(X, Y) Z=0 .
\end{aligned}
$$

Putting $X=\xi$ in (3.4) and then taking the inner product with $\xi$, we obtain

$$
\begin{aligned}
& g\left(P(\xi, Y) W_{2}(U, V) Z, \xi\right)-g\left(W_{2}(P(\xi, Y) U, V) Z, \xi\right) \\
& \quad-g\left(W_{2}(U, P(\xi, Y) V) Z, \xi\right)-g\left(W_{2}(U, V) P(\xi, Y) Z, \xi\right)=0 .
\end{aligned}
$$

Using (3.2) in (3.5), we obtain

$$
\begin{aligned}
-g\left(Y, W_{2}(U, V) Z\right)-g(Y, U) \eta\left(W_{2}(\xi, V) Z\right)-g(Y, V) \eta\left(W_{2}(U, \xi) Z\right)-g(Y, Z) \eta\left(W_{2}(U, V) \xi\right) \\
+\frac{1}{n-1}\left[S\left(Y, W_{2}(U, V) Z\right)+S(Y, U) \eta\left(W_{2}(\xi, V) Z\right)\right. \\
\left.+S(Y, V) \eta\left(W_{2}(U, \xi) Z\right)+S(Y, Z) \eta\left(W_{2}(U, V) \xi\right)\right]=0 .
\end{aligned}
$$

By using (2.16) in (3.6), we get

$$
g\left(Y, W_{2}(U, V) Z\right)-\frac{1}{n-1} S\left(Y, W_{2}(U, V) Z\right)=0 .
$$

Taking $U=Z=\xi$ in (3.7) and using (2.11) and (2.6), we have

$$
S(Q Y, V)=2(n-1) S(Y, V)-(n-1)^{2} g(Y, V) .
$$


This implies that

$$
Q Y=(n-1) Y .
$$

From this, we get

$$
S(Y, V)=(n-1) g(Y, V) .
$$

Thus, we can state the following.

Theorem 3.1. An LP-Sasakian manifold $M$ satisfying the condition $P(X, Y) \cdot W_{2}=0$ is an Einstein manifold.

\section{LP-Sasakian Manifold Satisfying $\widetilde{M}(X, Y) \cdot W_{2}=0$}

The $M$-projective curvature tensor $\widetilde{M}$ is defined as [11]

$$
\begin{aligned}
\widetilde{M}(X, Y) Z=R(X, Y) Z-\frac{1}{2(n-1)}[ & S(Y, Z) X-S(X, Z) Y \\
& +g(Y, Z) Q X-g(X, Z) Q Y]
\end{aligned}
$$

Using (2.6) and (2.8), (4.1) reduces to

$$
\widetilde{M}(\xi, Y) Z=\frac{1}{2}[g(Y, Z) \xi-\eta(Z) Y]-\frac{1}{2(n-1)}[S(Y, Z) \xi-\eta(Z) Q Y]
$$

Suppose that in an LP-Sasakian manifold

$$
\widetilde{M}(X, Y) \cdot W_{2}=0
$$

This condition implies that

$$
\begin{aligned}
& \widetilde{M}(X, Y) W_{2}(U, V) Z-W_{2}(\widetilde{M}(X, Y) U, V) Z \\
& \quad-W_{2}(U, \widetilde{M}(X, Y) V) Z-W_{2}(U, V) \widetilde{M}(X, Y) Z=0 .
\end{aligned}
$$

Putting $X=\xi$ in (4.4) and then taking the inner product with $\xi$, we obtain

$$
\begin{aligned}
& g\left(\widetilde{M}(\xi, Y) W_{2}(U, V) Z, \xi\right)-g\left(W_{2}(\widetilde{M}(\xi, Y) U, V) Z, \xi\right) \\
& \quad-g\left(W_{2}(U, \widetilde{M}(\xi, Y) V) Z, \xi\right)-g\left(W_{2}(U, V) \widetilde{M}(\xi, Y) Z, \xi\right)=0 .
\end{aligned}
$$


Using (4.2) in (4.5), we obtain

$$
\begin{aligned}
\frac{1}{2}\left[-g\left(Y, W_{2}(U, V) Z\right)-g(Y, U) \eta\left(W_{2}(\xi, V) Z\right)-g(Y, V) \eta\left(W_{2}(U, \xi) Z\right)\right. \\
-g(Y, Z) \eta\left(W_{2}(U, V) \xi\right)+\eta(U) \eta\left(W_{2}(Y, V) Z\right)+\eta(V) \eta\left(W_{2}(U, Y) Z\right) \\
\left.+\eta(Z) \eta\left(W_{2}(U, V) Y\right)\right] \\
+\frac{1}{2(n-1)}\left[S\left(Y, W_{2}(U, V) Z\right)+S(Y, U) \eta\left(W_{2}(\xi, V) Z\right)\right. \\
+S(Y, V) \eta\left(W_{2}(U, \xi) Z\right)+S(Y, Z) \eta\left(W_{2}(U, V) \xi\right)-\eta(U) \eta\left(W_{2}(Q Y, V) Z\right) \\
\left.-\eta(V) \eta\left(W_{2}(U, Q Y) Z\right)-\eta(Z) \eta\left(W_{2}(U, V) Q Y\right)\right]=0 .
\end{aligned}
$$

By using (2.16) in (4.6), we get

$$
\frac{1}{2} g\left(Y, W_{2}(U, V) Z\right)-\frac{1}{2(n-1)} S\left(Y, W_{2}(U, V) Z\right)=0 .
$$

Taking $U=Z=\xi$ in (4.7) and using (2.11) and (2.6), we have

$$
S(Q Y, V)=2(n-1) S(Y, V)-(n-1)^{2} g(Y, V)
$$

This implies that

$$
Q Y=(n-1) Y,
$$

which gives

$$
S(Y, V)=(n-1) g(Y, V)
$$

Thus, we can state the following.

Theorem 4.1. An LP-Sasakian manifold $M$ satisfying the condition $\widetilde{M}(X, Y) \cdot W_{2}=0$ is an Einstein manifold.

\section{LP-Sasakian Manifolds Satisfying $C(X, Y) \cdot W_{2}=0$}

The conformal curvature tensor $C$ is defined as [12]

$$
\begin{aligned}
C(X, Y) Z= & R(X, Y) Z-\frac{1}{n-2}[S(Y, Z) X-S(X, Z) Y+g(Y, Z) Q X-g(X, Z) Q Y] \\
& +\frac{r}{(n-1)(n-2)}[g(Y, Z) X-g(X, Z) Y]
\end{aligned}
$$


Using (2.6) and (2.8), (5.1) reduces to

$$
C(\xi, Y) Z=\frac{1-n+r}{(n-1)(n-2)}[g(Y, Z) \xi-\eta(Z) Y]-\frac{1}{n-2}[S(Y, Z) \xi-\eta(Z) Q Y]
$$

Now consider an LP-Sasakian manifold satisfying

$$
C(X, Y) \cdot W_{2}=0
$$

This condition implies that

$$
\begin{aligned}
& C(X, Y) W_{2}(U, V) Z-W_{2}(C(X, Y) U, V) Z \\
& \quad-W_{2}(U, C(X, Y) V) Z-W_{2}(U, V) C(X, Y) Z=0 .
\end{aligned}
$$

Putting $X=\xi$ in (5.4) and then taking the inner product with $\xi$, we obtain

$$
\begin{aligned}
& g\left(C(\xi, Y) W_{2}(U, V) Z, \xi\right)-g\left(W_{2}(C(\xi, Y) U, V) Z, \xi\right) \\
& \quad-g\left(W_{2}(U, C(\xi, Y) V) Z, \xi\right)-g\left(W_{2}(U, V) C(\xi, Y) Z, \xi\right)=0 .
\end{aligned}
$$

Using (5.2) in (5.5), we obtain

$$
\begin{aligned}
\frac{1-n+r}{(n-1)(n-2)}\left[-g\left(Y, W_{2}(U, V) Z\right)-g(Y, U) \eta\left(W_{2}(\xi, V) Z\right)-g(Y, V) \eta\left(W_{2}(U, \xi) Z\right)\right. \\
-g(Y, Z) \eta\left(W_{2}(U, V) \xi\right)-\eta(Y) \eta\left(W_{2}(U, V) Z\right)+\eta(U) \eta\left(W_{2}(Y, V) Z\right) \\
\left.+\eta(V) \eta\left(W_{2}(U, Y) Z\right)+\eta(Z) \eta\left(W_{2}(U, V) Y\right)\right] \\
+\frac{1}{n-2}\left[S\left(Y, W_{2}(U, V) Z\right)+S(Y, U) \eta\left(W_{2}(\xi, V) Z\right)+S(Y, V) \eta\left(W_{2}(U, \xi) Z\right)\right. \\
+S(Y, Z) \eta\left(W_{2}(U, V) \xi\right)+(n-1) \eta(Y) \eta\left(W_{2}(U, V) Z\right) \\
-\eta(U) \eta\left(W_{2}(Q Y, V) Z\right) \\
\left.-\eta(V) \eta\left(W_{2}(U, Q Y) Z\right)-\eta(Z) \eta\left(W_{2}(U, V) Q Y\right)\right]=0 .
\end{aligned}
$$

By using (2.16) in (5.6), we get

$$
\left\{\frac{1-n+r}{(n-1)(n-2)}\right\} g\left(Y, W_{2}(U, V) Z\right)-\frac{1}{n-2} S\left(Y, W_{2}(U, V) Z\right)=0 .
$$

Taking $U=Z=\xi$ in (5.7) and then using (2.11) and (2.6), we have

$$
S(Q Y, V)=\left\{\frac{2+n(n-3)+r}{n-1}\right\} S(Y, V)-(n-1+r) g(Y, V) .
$$


This implies that

$$
Q Y=(n-1) Y,
$$

and it follows that

$$
S(Y, V)=(n-1) g(Y, V) .
$$

Thus, we can state the following.

Theorem 5.1. An LP-Sasakian manifold $M$ satisfying the condition $C(X, Y) \cdot W_{2}=0$ is an Einstein manifold.

\section{Acknowledgment}

The authors express their thanks to DST (Department of Science and Technology), Government of India for providing financial assistance under major research project.

\section{References}

[1] K. Matsumoto, "On Lorentzian paracontact manifolds," Bulletin of the Yamagata University. Natural Science, vol. 12, no. 2, pp. 151-156, 1989.

[2] I. Mihai and R. Roşca, "On Lorentzian P-Sasakian manifolds," in Classical Analysis (Kazimierz Dolny, 1991), pp. 155-169, World Scientific, River Edge, NJ, USA, 1992.

[3] K. Matsumoto and I. Mihai, "On a certain transformation in a Lorentzian para-Sasakian manifold," Tensor, vol. 47, no. 2, pp. 189-197, 1988.

[4] I. Mihai, A. A. Shaikh, and U. C. De, “On Lorentzian para-Sasakian manifolds," Rendiconti del Seminario Matematico di Messina, no. 3, pp. 149-158, 1999.

[5] Venkatesha and C. S. Bagewadi, “On concircular $\phi$-recurrent LP-Sasakian manifolds," Differential Geometry-Dynamical Systems, vol. 10, pp. 312-319, 2008.

[6] G. P. Pokhariyal and R. S. Mishra, "Curvature tensors' and their relativistics significance," Yokohama Mathematical Journal, vol. 18, pp. 105-108, 1970.

[7] G. P. Pokhariyal, "Study of a new curvature tensor in a Sasakian manifold," Tensor, vol. 36, no. 2, pp. 222-226, 1982.

[8] K. Matsumoto, S. Ianuş, and I. Mihai, "On P-Sasakian manifolds which admit certain tensor fields," Publicationes Mathematicae Debrecen, vol. 33, no. 3-4, pp. 199-204, 1986.

[9] A. Yíldíz and U. C. De, "On a type of Kenmotsu manifolds," Differential Geometry-Dynamical Systems, vol. 12, pp. 289-298, 2010.

[10] K. Yano and M. Kon, Structures on Manifolds, vol. 3 of Series in Pure Mathematics, World Scientific, Singapore, 1984.

[11] G. P. Pokhariyal and R. S. Mishra, "Curvature tensors and their relativistic significance. II," Yokohama Mathematical Journal, vol. 19, no. 2, pp. 97-103, 1971.

[12] L. P. Eisenhart, Riemannian Geometry, Princeton University Press, Princeton, NJ, USA, 1949. 


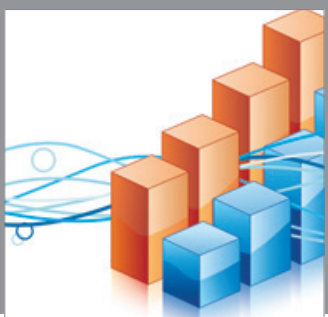

Advances in

Operations Research

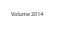

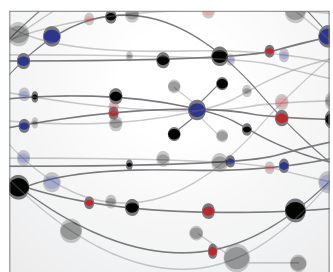

\section{The Scientific} World Journal
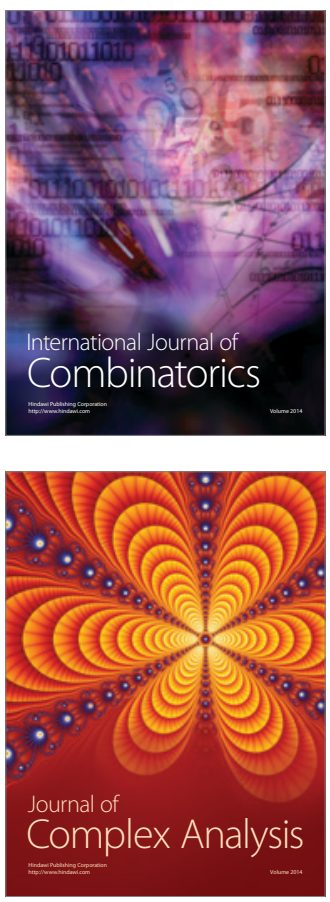

International Journal of

Mathematics and

Mathematical

Sciences
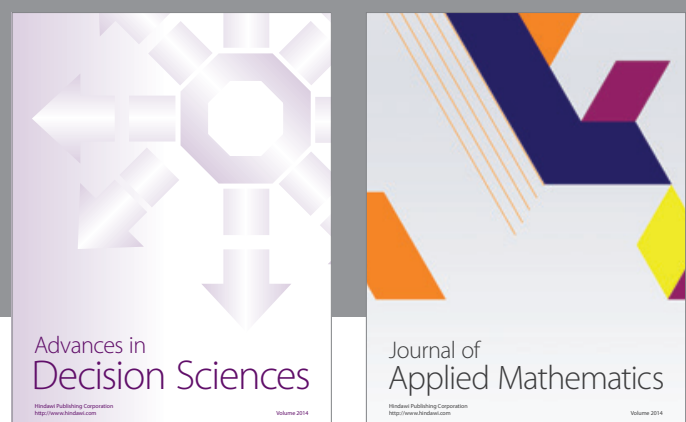

Journal of

Applied Mathematics
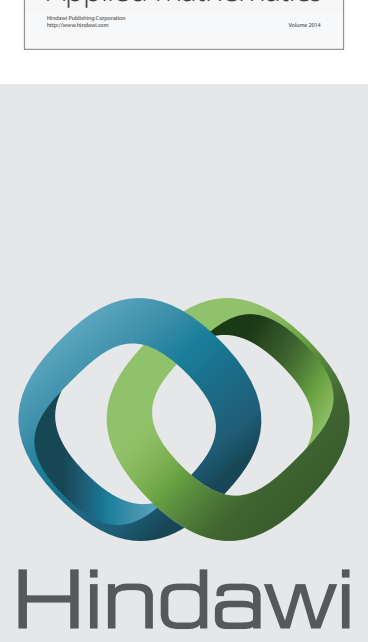

Submit your manuscripts at http://www.hindawi.com
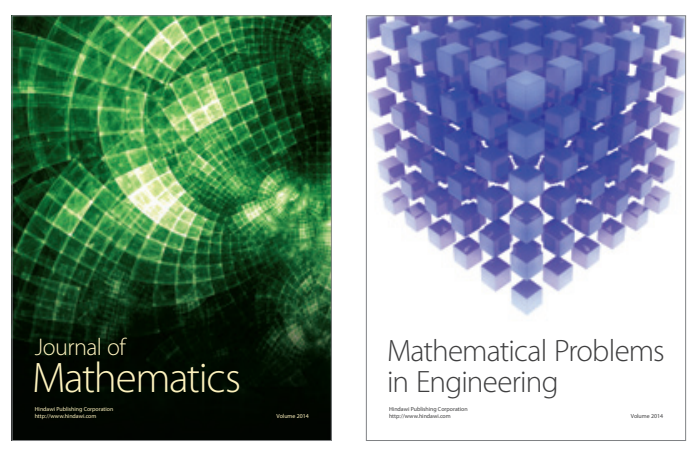

Mathematical Problems in Engineering
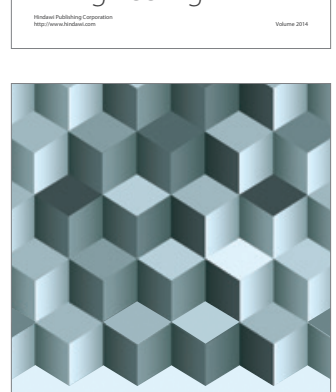

Journal of

Function Spaces
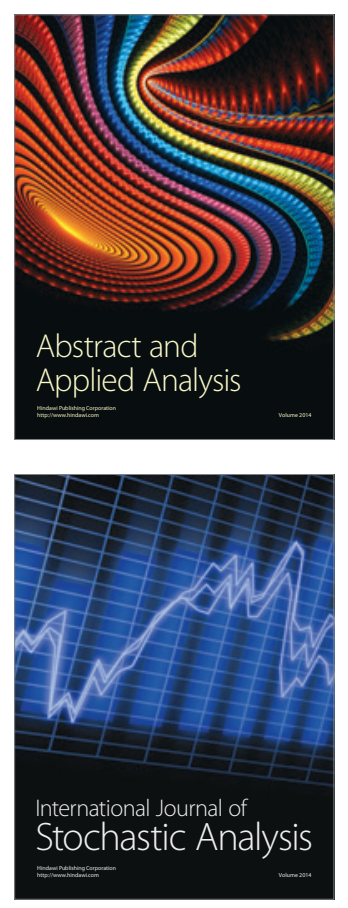

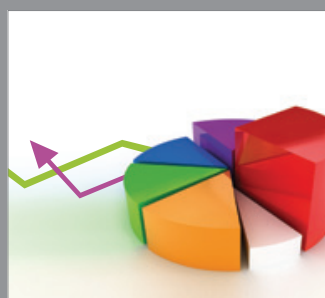

ournal of

Probability and Statistics

Promensencen
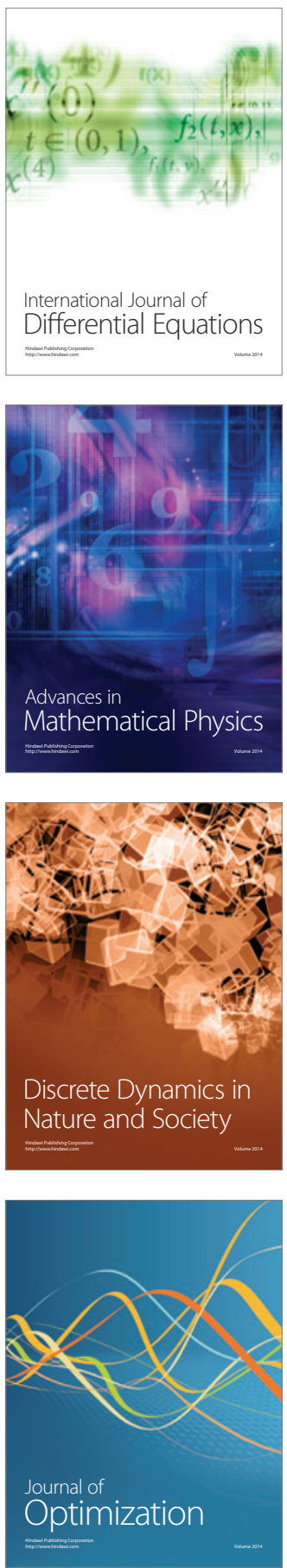